\title{
Burden of Hypertension in Recently Diagnosed Type 2 Diabetes Mellitus
}

\section{Sharma SK, Karki P, Shrestha N, Dhakal S, Gupta PR Ahmed W S, Gupta N, Badu B, Rajbhandari S,}

Cardiovascular diseases are the major cause of death In diabetes patients. Many factors including hypertension contribute to the high prevalence of cardiovascular disease in diabetes.

A total of 111 persons with recently diagnosed Type 2 diabetes mellitus were analysed forthe association of hypertension at the time of presentation to diabetes clinic of B P Koirala Institute of Health Sciences, Dharan, Nepal. Majority of the patients belonged to age group of 40 to 60 years. 56 (50\%) of them were hypertensive by JNC VII criteria. Body Mass Index (BMI) of $>25$ were found in 71 persons, however association of Hypertension with various categories of BMI was statistically not significant. Hypertension was more common in overt proteinuric diabetes $(59 \%)$ than person with microalbuminuria and nonalbuminuria. Half of the recently diagnosed Type 2 Diabetes were hypertensive. Majority of them also had proteinuria, a surrogate marker of cardiovascular events. Community education and surveillance is essential for early detection and prevention of cardiovascular risk factors in persons with diabetes.

Diabetes Clinic, Dapartment of Medicine, BP Koiraia institute of Health Sciences, Dharan, Nepal, 